COMMUNICATIONS IN

ANALYSIS AND GEOMETRY

Volume 12, Number 1, 93-109, 2004

\title{
Maps With Prescribed Tension Fields
}

\author{
Wenyi Chen AND JÜrgen Jost
}

We consider maps into a Riemannian manifold of nonpositive sectional curvature with prescribed tension field. We derive a priori estimates and solve a Dirichlet problem.

\section{Introduction.}

Elliptic regularity theory is traditionally concerned with functions $f: \Omega \rightarrow$ $\mathbb{R}, \Omega$ being some domain in some Euclidean space $\mathbb{R}^{m}$, or, more generally, in a Riemannian manifold $M . f$ is assumed to solve some elliptic PDE, and the regularity theory derives estimates of various norms of $f$ in terms of some data (boundary conditions, geometry of the domain) and some structural constants of the elliptic operator. The prototype here is the Laplace operator, and the elliptic equation in the simplest case then reduces to the Poisson equation

$$
\Delta f=v,
$$

for some prescribed $v$, plus some boundary condition. The idea of elliptic regularity theory then is to control some Sobolev or Hölder norm of $f$,

$$
\|f\|_{W^{k, p}}, \text { or }\|f\|_{C^{k, \alpha}}
$$

in terms of the corresponding norm of $\Delta f$ for $k-2$ in place of $k$ plus some terms depending on the data. This may then be applied to estimate a solution of the Poisson equation through the prescribed right hand side $v$. As is well-known, such estimates provide the basis for the existence theory for solutions of elliptic equations and a guide for the construction of numerical approximation schemes (for a detailed presentation, see e.g. [5]).

We are interested here not in functions, but in maps $f: \Omega \rightarrow N$ into some Riemannian manifold $N$. This will make the problem genuinely nonlinear. Nevertheless, the Laplace operator $\Delta f$ naturally generalizes to the tension field $\tau(f)$, given in local coordinates by

$$
\tau(f)^{k}=\frac{1}{\sqrt{\gamma(x)}} \frac{\partial}{\partial x^{\alpha}}\left(\gamma^{\alpha \beta}(x) \sqrt{\gamma(x)} \frac{\partial f^{k}(x)}{\partial x^{\beta}}\right)+\gamma^{\alpha \beta}(x) \Gamma_{j k}^{i}(f(x)) \frac{\partial f^{i}(x)}{\partial x^{\alpha}} \frac{\partial f^{j}(x)}{\partial x^{\beta}},
$$


with the standard notation:

$\left(\gamma_{\alpha \beta}(x)\right)_{\alpha, \beta=1, \ldots, m}$ is the Riemannian metric tensor on the domain $\Omega \subset M$ w.r.t. local coordinates $x=\left(x^{1}, \ldots, x^{m}\right),\left(\gamma^{\alpha \beta}\right)_{\alpha, \beta=1, \ldots, m}$ its inverse, $\gamma=$ $\operatorname{det}\left(\gamma_{\alpha \beta}\right) . \Gamma_{j k}^{i}(f)$ are the Christoffel symbols of the Riemannian metric tensor $\left(g_{i j}(f)\right)_{i, j=1, \ldots, n}$ on $N$, where we take the liberty to identify $f$ with its local coordinate representation $\left(f^{1}, \ldots, f^{n}\right)$.

Those local coordinates can be chosen in such a manner that the Christoffel symbols $\Gamma_{j k}^{i}(f)$ vanish precisely if $N$ is flat, and in that case, the tension field reduces to the Laplacian of the domain $\Omega$. In general, however, a Riemannian manifold is not flat, and so the tension field then is a nonlinear elliptic operator.

In more abstract terms, $\tau(f)$ is a section of the bundle $f^{-1} T N$, and therefore fundamentally nonlinear as this bundle itself depends on the map $f$.

This tension field is the negative gradient field of the energy functional

$E(f)=\frac{1}{2} \int|d f(x)|^{2} \operatorname{dvol}(M)=\frac{1}{2} \int \gamma^{\alpha \beta}(x) g_{i j}(f(x)) \frac{\partial f^{i}}{\partial x^{\alpha}} \frac{\partial f^{j}}{\partial x^{\beta}} \sqrt{\gamma(x)} d x^{1} \ldots d x^{m}$

in the same way that the Laplace operator is the negative gradient field of the Dirichlet integral. Critical points of the energy integral, i.e. solutions of

$$
\tau(f)=0,
$$

are called harmonic maps and have been intensively studied.

More generally, given some function $\Phi: N \rightarrow \mathbb{R}$, one may look for critical points of the integral

$$
E_{\Phi}(f):=\frac{1}{2} \int|d f(x)|^{2} \operatorname{dvol}(M)+\int \Phi(f(x)) \operatorname{dvol}(M) .
$$

Solutions solve a system of type

$$
\tau(f)=V(f),
$$

for some vector field $V$ on $N$, and are called harmonic maps with potential, see e.g. [1], [9], [3].

In the present paper, we shall be concerned with the system

$$
\tau(f(x))=V(x, f(x))
$$

for some given $V$, without assuming a variational structure, however. Since $\tau(f)$ is a section of $f^{-1} T N$, also the right hand side naturally has to depend 
on $f$, in contrast to the linear Poisson equation where $V$ is a function of $x$ only.

The existence problem has been studied by von Wahl [11] in case of a domain $\Omega$ with boundary and prescribed boundary values, and by Jost-Yau [6] for this case as well as for the more subtle one of a closed manifold $M$. In particular, it turns out that, without a variational structure, on a closed manifold $M$, the above problem need not possess a solution. This is in contrast to the Dirichlet problem on $\Omega$ that has a solution under general circumstances, as shown in those papers.

Actually, all these results need some curvature assumptions on the target $N$, and, without imposing a size restriction, one needs the assumption that $N$ have nonpositive sectional curvature. Therefore, we shall assume throughout this paper that $N$ has nonpositive sectional curvature. Under this assumption, we wish to study the above problem as a nonlinear generalization of the Poisson equation. Still, while we no longer have a linear structure, the curvature assumption implies that there exists an underlying convex geometry as has been explored in more abstract terms in [4].

The first part of our paper is concerned with extending elliptic regularity theory to the present nonlinear setting. The guiding idea is that the tension field $\tau$ should assume the role of the Laplacian $\Delta$. An additional feature, however, is that not only the geometry is nonlinear, but also that the topology is nontrivial. Therefore, our estimates will also involve a topological datum, namely the homotopy class of the map in question. With that addition, we are able to generalize the fundamental elliptic $W^{2,2}$ estimate to our setting. In Theorem. 2.2, we shall estimate the $L^{2}$-norms of the first and second derivatives of any map $f: M \rightarrow N$ solely in terms of the $L^{2}$-norm of its tension field plus a topological term that only depends on the homotopy class $[f]$ of $f$.

Of course, the constants in those estimates will depend on the underlying geometry, in particular on a bound for the Ricci curvature of $M$.

In the second part of our paper, we study the Dirichlet problem for

$$
\tau(f)=V
$$

Our result will need some bound on $V$ depending on the first eigenvalue of $\Omega$. The result as well as the method are different from those obtained by von Wahl and Jost-Yau. 


\section{A nonlinear $W^{2,2}-$ estimate.}

A continuous map $f: M: \rightarrow N$ induces a homeomorphism

$$
\rho=f_{\#}: \pi_{1}(M) \rightarrow \pi_{1}(N)
$$

of fundamental groups and at the same time a lift

$$
\widetilde{f}: \widetilde{M} \rightarrow \widetilde{N}
$$

We shall need the $\rho$-equivariance of the lift map $\widetilde{f}$, i.e.

$$
\widetilde{f}(\lambda x)=\rho(\lambda) \tilde{f}(x)
$$

for all $x \in \widetilde{M}, \lambda \in \pi_{1}(M)$ where the fundamental groups $\pi_{1}(M)$ and $\pi_{1}(N)$ act isometrically on $\widetilde{M}$ and $\widetilde{N}$ by deck transformations respectively so that $M=\widetilde{M} / \pi_{1}(M)$ and $N=\widetilde{N} / \pi_{1}(N)$. There is a correspondence between the $\rho$-equivariant maps from $\widetilde{M}$ to $\widetilde{N}$ and maps from $M$ to $N$.

For the complete simply connected Riemannian manifold of nonpositive sectional curvature $\tilde{N}$, the distance function

$$
d: \widetilde{N} \times \widetilde{N} \rightarrow \mathbb{R}, \quad d(u, v)=\text { the distance from } u \text { to } v
$$

is well defined and we have a smooth function $d^{2}$ on the manifold $\widetilde{N} \times \widetilde{N}$. Let $f_{1}, f_{2}: \widetilde{M} \rightarrow \widetilde{N}$ be $\rho$-equivariant maps, then the function $d^{2}\left(f_{1}, f_{2}\right)$ is also smooth on the manifold $M$ because deck transformations are isometric. For $(u, v) \in \widetilde{N} \times \widetilde{N}$, we choose an orthonormal basis $e_{1}, e_{2}, \cdots, e_{n}$ for $T_{u} \widetilde{N}$. By parallel translation along the shortest geodesic from $u$ to $v$ on $\widetilde{N}$, we get a basis $\bar{e}_{1}, \bar{e}_{2}, \cdots, \bar{e}_{n}$ for $T_{v} \widetilde{N}$. Take $e_{1}, e_{2}, \cdots, e_{n}, \bar{e}_{1}, \bar{e}_{2}, \cdots, \bar{e}_{n}$ as a local orthonomal frame for $T_{(u, v)} N \times N$. Let $\theta^{1}, \theta^{2}, \cdots, \theta^{m}$ be an orthonormal coframe in a neighbourhood of $x \in \widetilde{M}$, we then write the differentials of the maps $f_{1}, f_{2}: \widetilde{M} \rightarrow \widetilde{N}$ as $d f_{1}=f_{1 \alpha}^{i} e_{i} \otimes \theta^{\alpha}$, and $d f_{2}=f_{2 \alpha}^{\bar{i}} \bar{e}_{i} \otimes \theta^{\alpha}$. We use the Einstein summation convention.

Now we define $E\left(f_{1}, f_{2}\right)$, the energy of the difference of the two maps $f_{1}$, $f_{2}$ by

$$
E\left(f_{1}, f_{2}\right)=\frac{1}{2} \int_{M} \sum_{i=1}^{n} \sum_{\alpha=1}^{m}\left(f_{1 \alpha}^{i}-f_{2 \alpha}^{\bar{i}}\right)^{2} d \operatorname{vol}(M)
$$

where the integral is calculated on a fundamental domain of $M$ in $\widetilde{M}$. Since parallel transport is isometric, we obtain a triangle inequality

$$
\left|\sqrt{E\left(f_{1}, f_{3}\right)}-\sqrt{E\left(f_{3}, f_{2}\right)}\right| \leq \sqrt{E\left(f_{1}, f_{2}\right)},
$$


Inparticular, the energy of the difference is symmetric, and for the energy of a map $f$,

$$
E(f)=\frac{1}{2} \int_{M} \sum_{i=1}^{n} \sum_{\alpha=1}^{m}\left(f_{\alpha}^{i}\right)^{2} d \operatorname{vol}(M)
$$

we then have

$$
\left|\sqrt{E\left(f_{1}\right)}-\sqrt{E\left(f_{2}\right)}\right| \leq \sqrt{E\left(f_{1}, f_{2}\right)}
$$

Putting $X_{\alpha}=f_{1 \alpha}^{i} e_{i}+f_{2 \alpha}^{\bar{i}} \bar{e}_{i}$, we then are able to express the Laplacian of $d^{2}\left(f_{1}, f_{2}\right)$ as

$$
\Delta d^{2}\left(f_{1}, f_{2}\right)=\left(d^{2}\right)_{X_{\alpha} X_{\alpha}}+d\left\{d_{i} f_{1 \alpha \alpha}^{i}+d_{\bar{i}} f_{2 \alpha \alpha}^{\bar{i}}\right\}
$$

where $\tau\left(f_{1}\right)^{1}=f_{1 \alpha \alpha}^{i}$ and $\tau\left(f_{1}\right)^{2}=f_{2 \alpha \alpha}^{\bar{i}}$ are the components of the tension fields of the maps $f_{1}, f_{2}$ respectively. It was shown in [10] that if $K_{N} \leq 0$ then

$$
\left(d^{2}\right)_{X_{\alpha} X_{\alpha}} \geq 2 \sum_{i}\left(f_{1 \alpha}^{i}-f_{2 \alpha}^{\bar{i}}\right)^{2} .
$$

Hence

$$
\Delta d^{2}\left(f_{1}, f_{2}\right) \geq 2 \sum_{i}\left(f_{1 \alpha}^{i}-f_{2 \alpha}^{\bar{i}}\right)^{2}-d\left\{\left|\tau\left(f_{1}\right)\right|+\left|\tau\left(f_{2}\right)\right|\right\} .
$$

We have also

$$
\Delta d\left(f_{1}, f_{2}\right) \geq-\left\{\left|\tau\left(f_{1}\right)\right|+\left|\tau\left(f_{2}\right)\right|\right\} .
$$

Let $f_{1}$ and $f_{2}$ be maps from a domain $\Omega$ of the manifold $M$, smooth enough in $\Omega$ and on the boundary $\partial \Omega$. If two maps $f_{1}$ and $f_{2}$ coincide on the boundary $\partial \Omega$, we then have by $(2.2)$

$$
\int_{\Omega}\left|\nabla d\left(f_{1}, f_{2}\right)\right|^{2} d v o l \leq\left\|d\left(f_{1}, f_{2}\right)\right\|_{L^{2}(\Omega)}\left\{\left\|\tau\left(f_{1}\right)\right\|_{L^{2}(\Omega)}+\left\|\tau\left(f_{2}\right)\right\|_{L^{2}(\Omega)}\right\} .
$$

Therefore

$$
\left\|d\left(f_{1}, f_{2}\right)\right\|_{L^{2}(\Omega)} \leq \lambda(\Omega)^{-1}\left\{\left\|\tau\left(f_{1}\right)\right\|_{L^{2}(\Omega)}+\left\|\tau\left(f_{2}\right)\right\|_{L^{2}(\Omega)}\right\}
$$

where the constant $\lambda(\Omega)$ is the first eigenvalue of the Laplacian on $\Omega$, see [2].

On the other hand, the integral of (2.1) over $\Omega$ gives

$$
E\left(f_{1}, f_{2}\right) \leq 2^{-1} \int_{\Omega} \Delta d^{2}\left(f_{1}, f_{2}\right)+d\left(f_{1}, f_{2}\right)\left\{\left|\tau\left(f_{1}\right)\right|+\left|\tau\left(f_{2}\right)\right|\right\} .
$$


Notice that $d^{2}\left(f_{1}, f_{2}\right) \geq 0$ in $\Omega$ and $d^{2}\left(f_{1}, f_{2}\right)=0$ on $\partial \Omega$, the derivative of $d^{2}\left(f_{1}, f_{2}\right)$ along the outer normal vector of $\Omega$ at $\partial \Omega$ will be nonpositive. Hence the Stokes formula gives

$$
\int_{\Omega} \Delta d^{2}\left(f_{1}, f_{2}\right) \leq 0 .
$$

We then obtain an energy estimate for two maps that have the same boundary values and belong to the same homotopy class, with (2.3)

$$
E\left(f_{1}, f_{2}\right) \leq \lambda(\Omega)^{-1}\left\{\left\|\tau\left(f_{1}\right)\right\|_{L^{2}(\Omega)}^{2}+\left\|\tau\left(f_{2}\right)\right\|_{L^{2}(\Omega)}^{2}\right\}
$$

For the case of a compact manifold $M$ without boundary, we need a deeper analysis. Integrating the inequality (2.1) over a fundamental domain, we get

$$
E\left(f_{1}, f_{2}\right) \leq \frac{1}{4}\left\|d\left(f_{1}, f_{2}\right)\right\|_{L^{2}(M)}\left\{\left\|\tau\left(f_{1}\right)\right\|_{L^{2}(M)}+\left\|\tau\left(f_{2}\right)\right\|_{L^{2}(M)}\right\} .
$$

A direct corollary of the triangle inequality and the inequality (2.5) is that if $h_{1}, h_{2}$ are two homotopic harmonic maps, then $E\left(h_{1}, h_{2}\right)=0$ and $E\left(f, h_{1}\right)=E\left(f, h_{2}\right)$ whenever $[f]=\left[h_{1}\right]$.

Lemma 2.1: Let $f_{0}, f_{1}: \widetilde{M} \rightarrow \widetilde{N}$ be $\rho$-equivariant maps. Define $f_{t}: \widetilde{M} \rightarrow$ $\widetilde{N}$ by exponential map

$$
f_{t}(x)=\exp _{f_{0}(x)}\left\{t \exp _{f_{0}(x)}^{-1} f_{1}(x)\right\}
$$

then $f_{t}$ is also $\rho$-equivariant and $\sqrt{E\left(f_{t}\right)}$ is a convex function of $t$.

Proof: Let $\gamma_{x}$ be the geodesic on $\widetilde{N}$ from $f_{0}(x)$ to $f_{1}(x)$, then $\gamma_{x}$ is also $\rho$-equivariant. Hence, $f_{t}$ is $\rho$-equivariant. Fix $\alpha \in\{1,2, \cdots, n\}$, we claim that $\nabla_{\alpha} f_{t}(x)$ is a Jacobi field along the geodesic $\gamma_{x}$. In fact, let $\nu_{\alpha} \in T_{x} \widetilde{M}$ be the vector dual to $\theta^{\alpha}, c(s)$ be the geodesic on $\widetilde{M}$ with $c(0)=x, c^{\prime}(0)=\nu_{\alpha}$, $C:(0,1) \times(-\delta, \delta) \rightarrow \widetilde{N}, C(t, s)=\exp _{f_{0}(c(s))}\left\{t \exp _{f_{0}(c(s))}^{-1} f_{1}(c(s))\right\}$, then for any fixed $s, C(\cdot, s)$ is a geodesic. On the other hand

$$
\nabla_{\alpha} f_{t}(x)=\left.\frac{\partial}{\partial s} C(t, s)\right|_{s=0}
$$


By the Jacobi field equation, we have

$$
\begin{aligned}
\frac{\partial^{2}}{\partial t^{2}} \sqrt{E\left(f_{t}\right)} & =\frac{-1}{\sqrt{E\left(f_{t}\right)}} \int_{M}\left\langle R\left(\nabla_{\alpha} f_{t}(x), \dot{\gamma}\right) \dot{\gamma}, \nabla_{\alpha} f_{t}(x)\right\rangle \operatorname{dvol}(M) \\
& +\frac{1}{\sqrt{E\left(f_{t}\right)}} \int_{M} \sum_{\alpha}\left|\frac{\partial}{\partial t} \nabla_{\alpha} f_{t}(x)\right|^{2} d \operatorname{vol}(M) \\
& -\frac{1}{E\left(f_{t}\right)^{\frac{3}{2}}}\left|\int_{M} \nabla_{\alpha} f_{t}(x), \nabla_{\alpha} \frac{\partial}{\partial t} f_{t}(x) d \operatorname{vol}(M)\right|^{2} \\
\geq & 0 .
\end{aligned}
$$

As required.

Lemma 2.2: There exists a constant $C$ which depends only on the homotopy class $[f]$ such that

$$
\|d(f, h)\|_{L^{2}(M)}^{2} \leq C E(f, h)
$$

holds for a harmonic map in the homotopy class $[f]$.

Proof: We prove the Lemma by contradiction. If there were a homotopy class such that the inequality (2.6) did not hold, we then could find a sequence $f_{k}$ of maps and harmonic maps $h_{k}$ in the same homotopy class such that

$$
\left\|d\left(f_{k}, h_{k}\right)\right\|_{L^{2}(M)}^{2}=\inf _{\tau(h)=0}\left\|d\left(f_{k}, h\right)\right\|_{L^{2}(M)}^{2} \geq k^{2} E\left(f_{k}, h_{k}\right)
$$

with $\left\|d\left(f_{k}, h\right)\right\|_{L^{2}(M)}^{2} \geq 1$. Notice that the energy of the difference $E\left(f_{k}, h\right)$ is independent of the choice of the harmonic map $h$. Define

$$
f_{k}^{t}(x)=\exp _{h_{k}(x)}\left\{t \exp _{h_{k}(x)}^{-1} f_{k}(x)\right\} .
$$

We have

$$
\begin{aligned}
\left\|d\left(f_{k}^{t}, h_{k}\right)\right\|_{L^{2}(M)}^{2} & =t^{2}\left\|d\left(f_{k}, h_{k}\right)\right\|_{L^{2}(M)}^{2} \geq k^{2} t^{2} E\left(f_{k}, h_{k}\right) \\
& \geq k^{2} t^{2}\left(\sqrt{E\left(f_{k}\right)}-\sqrt{E\left(h_{k}\right)}\right)^{2}
\end{aligned}
$$

It follows from the convexity of the energy $E\left(f_{k}^{t}\right)$ that

$$
\left\|d\left(f_{k}^{t}, h_{k}\right)\right\|_{L^{2}(M)} \geq k\left|\sqrt{E\left(f_{k}\right)}-\sqrt{E\left(h_{k}\right)}\right| .
$$


Choose $t=t_{k}$ such that $\left\|d\left(f_{k}^{t}, h_{k}\right)\right\|_{L^{2}(M)}=1$. From (2.8), $f_{k}^{t_{k}}$ is a minimizing sequence for the energy. Therefore the sequence $f_{k}^{t_{k}}$ converges strongly to a harmonic map $h$, i.e.

$$
\left\|d\left(f_{k}^{t_{k}}, h\right)\right\|_{L^{2}(M)} \rightarrow 0
$$

Notice that

$$
\begin{aligned}
\left\|d\left(f_{k}, f_{k}^{t_{k}}\right)\right\|_{L^{2}(M)} & =\left\|d\left(f_{k}, h_{k}\right)-d\left(h_{k}, f_{k}^{t_{k}}\right)\right\|_{L^{2}(M)} \\
& =\left\|\left(1-t_{k}\right) d\left(f_{k}, h_{k}\right)\right\|_{L^{2}(M)} \\
& =\left\|d\left(f_{k}, h_{k}\right)\right\|_{L^{2}(M)}-t_{k}\left\|d\left(f_{k}, h_{k}\right)\right\|_{L^{2}(M)} \\
& =\left\|d\left(f_{k}, h_{k}\right)\right\|_{L^{2}(M)}-1 .
\end{aligned}
$$

Hence for $k$ large enough,

$$
\begin{aligned}
\left\|d\left(f_{k}, h\right)\right\|_{L^{2}(M)} & \leq\left\|d\left(f_{k}, f_{k}^{t_{k}}\right)\right\|_{L^{2}(M)}+\frac{1}{2} \\
& =\left\|d\left(f_{k}, h_{k}\right)\right\|_{L^{2}(M)}-\frac{1}{2} .
\end{aligned}
$$

This is a contradiction to the choice of the harmonic maps $h_{k}$. This proves that the inequality $(2.3)$ holds for $\inf _{\tau(h)=0}\|d(f, h)\|_{L^{2}(M)}^{2} \geq 1$. Notice that the harmonic map and the inequality (2.6) are invariant under the rescaling of the metric on $N$, the inequality (2.6) holds for all maps in the same homotopy class.

Now we summarize the above, that is, (2.0), (2.5) and (2.6) as:

Theorem 2.1: Let $M$ be a compact Riemanian manifold with or without boundary, $N$ a compact Riemannian manifold of nonpositive sectional curvature. For a given homotopy class of maps from $M$ to $N$, with prescibed boundary values when the boundary of $M$ is not empty, there is a constant $C$ such that for any map $f$ in this homotopy class there is a harmonic map $h$ in the homotopy class such that

$$
\left(\int_{M}|d f|^{2}\right)^{\frac{1}{2}} \leq\left(\int_{M}|d h|^{2}\right)^{\frac{1}{2}}+C\left(\int_{M}|\tau(f)|^{2}\right)^{\frac{1}{2}}
$$

where $\tau(f)$ is the tension field. 
The first term on the right hand side depends only on the homotopy class of $f$, because all harmonic maps in the same homotopy class have the same -minimal- energy.

Recall the Bochner formula ([6], Ch. 8)

$$
\begin{aligned}
\Delta e(f)(x)= & |\nabla d f|^{2}+\langle\Delta(d f), d f\rangle+\left\langle d f\left(\operatorname{Ric}^{M}\left(e_{\alpha}\right)\right), d f\left(e_{\alpha}\right)\right\rangle \\
& -\left\langle R^{N}\left(d f\left(e_{\alpha}\right), d f\left(e_{\beta}\right)\right) d f\left(e_{\beta}\right), d f\left(e_{\alpha}\right)\right\rangle .
\end{aligned}
$$

Integrating it on the domain $M$ and making use of the inequality (2.10) under the assumption of nonpositive curvature, we get the main result of this section, namely an estimate for the $L^{2}-$ norm of the first and second derivatives of a map in a given homotopy class in terms of its tension field.

Theorem 2.2: Let $M$ be a compact Riemanian manifold without boundary, $N$ a compact Riemannian manifold of nonpositive sectional curvature. For a given homotopy class of maps from $M$ to $N$, let $h$ be a harmonic map in that homotopy class. We then have, for any map $f$ in that homotopy class,

$$
\int_{M}|d f|^{2} d \operatorname{vol}(M)+\int_{M}|\nabla d f|^{2} d v o l(M) \leq C_{1} \int_{M}|\tau(f)|^{2} d \operatorname{vol}(M)+C_{2} \int_{M}|d h|^{2} d v o l(M)
$$

where the constant $C_{1}$ depends only on the homotopy class of the map $f$ and the constant $C_{2}$ is $1+\left\|R i c^{M}\right\|_{\infty}$, where Ric ${ }^{M}$ is the Ricci curvature of $M$.

We point out once more that the last term on this inequality depends only on the homotopy class (and on the geometry of $M$ and $N$ ), but not in any way on the map $f$ in that homotopy class that we are estimating here.

By differentiation, we may then also obtain higher order estimates in a standard manner.

\section{Boundary value problems.}

We now let $\Omega$ be a domain in a manifold $M$ with a nonempty boundary $\partial \Omega$ and compact closure $\bar{\Omega}$. For the moment, we assume that the map $g: \bar{\Omega} \rightarrow N$ is of class $C^{2, \alpha}$.

Consider the parabolic system

$$
\left\{\begin{array}{l}
\tau(f)-\frac{\partial f}{\partial t}=V(f) \\
f(x, 0)=g(x), \quad x \in \Omega \\
f(x, t)=g(x), \quad x \in \partial \Omega \\
f: \bar{\Omega} \times[0, \infty) \rightarrow N
\end{array}\right.
$$


Remark: If there is an underlying variational structure, as for harmonic maps with potential, namely,

$$
E_{\varphi}(f)=\frac{1}{2} \int_{\Omega}|d f|^{2} d \operatorname{vol}(M)+\int_{\Omega} \varphi(f(x)) \operatorname{dvol}(M),
$$

for some function $\varphi: N \rightarrow R$, we have the Euler-Lagrange equation as

$$
\tau(f)(x)=\nabla \varphi(f(x)) .
$$

We have

$$
\frac{\partial}{\partial t} E_{\varphi}(f(\cdot, t))=-\int_{\Omega}\left|\frac{\partial}{\partial t} f(x, t)\right|^{2} d \operatorname{vol}(M),
$$

for a solution of the associated parabolic problem

$$
\frac{\partial f}{\partial t}=\tau(f)-\nabla \varphi(f)
$$

and therefore, if we assume that $E_{\varphi}(f)$ is bounded from below, i.e.,

$$
E_{\varphi}(f) \geq C
$$

for some $C \in \mathbb{R}$ and all $f$, then $\frac{\partial}{\partial t} f(\cdot, t)$ subconverges to zero in $L^{2}$ for $t \rightarrow \infty$, and the analysis becomes easy. This has been explained in the literature, see e. g., [3]. Without a variational structure, however, the problem is more difficult.

The smoothness of the map $g$ and the theory of linear parabolic systems give us a short time solution of the parabolic problem (3.1), i.e., there is a positive $T$ so that there is a $C^{2, \alpha}$ solution on $\Omega \times[0, T)$. The condition we impose on the vector field is that

$$
\nabla V(X, X) \geq-\mu|X|^{2}, \quad X \in \Gamma(T \Omega) .
$$

We also let $\lambda(\Omega)$ be the first nontrivial eignvalue for the Dirichlet problem on $\Omega$.

Lemma 3.1: Let $f$ satisfy the parabolic system (3.1) where the vector field $V$ satisfies (3.2) with $\mu \leq \frac{3}{4} \lambda(\Omega)$, then

$$
\int_{\Omega}\left|\frac{\partial}{\partial t} f(\cdot, t)\right|^{4} \leq \int_{\Omega}|\tau(g)-V(g)|^{4} .
$$


Proof: A direct computation gives that

$$
\begin{aligned}
\left(\Delta-\frac{\partial}{\partial t}\right)\left\langle\frac{\partial f}{\partial t}, \frac{\partial f}{\partial t}\right\rangle= & 2 \nabla V\left(\frac{\partial f}{\partial t}, \frac{\partial f}{\partial t}\right)+2\left\langle\nabla_{\nu_{i}} \frac{\partial f}{\partial t}, \nabla_{\nu_{i}} \frac{\partial f}{\partial t}\right\rangle \\
& -2\left\langle R^{N}\left(f_{*} \nu_{i}, \frac{\partial f}{\partial t}\right) f_{*} \nu_{i}, \frac{\partial f}{\partial t}\right\rangle .
\end{aligned}
$$

With the assumptions of the Lemma and the nonpositivity of the sectional curvature of the manifold $N$, it follows from the above identity

$$
\left(\Delta-\frac{\partial}{\partial t}\right)\left|\frac{\partial f}{\partial t}\right|^{2} \geq-\frac{3}{2} \lambda(\Omega)\left|\frac{\partial f}{\partial t}\right|^{2}+2\left|\nabla \frac{\partial f}{\partial t}\right|^{2} .
$$

Notice that

$$
2\left|\nabla \frac{\partial f}{\partial t}\right|^{2} \cdot\left|\frac{\partial f}{\partial t}\right|^{2} \geq\left.\left.\frac{1}{2}|\nabla| \frac{\partial f}{\partial t}\right|^{2}\right|^{2}
$$

Multiplying the two sides of the inequality (3.4) by $\left|\frac{\partial f}{\partial t}\right|^{2}$ and then integrating over $\Omega$, one obtains

$$
-\frac{1}{2} \frac{\partial}{\partial t} \int_{\Omega}\left|\frac{\partial f}{\partial t}\right|^{4} \geq\left.\left.\frac{3}{2} \int_{\Omega}|\nabla| \frac{\partial f}{\partial t}\right|^{2}\right|^{2}-\frac{3}{2} \lambda(\Omega) \int_{\Omega}\left|\frac{\partial f}{\partial t}\right|^{4} .
$$

Because $\frac{\partial f}{\partial t}=0$ on the boundary of the domain $\Omega$, the right side of the above inequality is nonnegative. Hence

$$
\frac{\partial}{\partial t} \int_{\Omega}\left|\frac{\partial f}{\partial t}\right|^{4} \leq 0
$$

This proves Lemma 3.1.

Let us return to the inequality (3.5)

$$
\left(\Delta-\frac{\partial}{\partial t}\right)\left\langle\frac{\partial f}{\partial t}, \frac{\partial f}{\partial t}\right\rangle \geq-C\left\langle\frac{\partial f}{\partial t}, \frac{\partial f}{\partial t}\right\rangle .
$$

Set $\psi(x, t)=\exp \{C t\}\left\langle\frac{\partial f}{\partial t}, \frac{\partial f}{\partial t}\right\rangle$, then

$$
\left(\Delta-\frac{\partial}{\partial t}\right) \psi \geq 0
$$

and $\psi=0$ on the boundary of $\Omega$. By Moser iteration (cf [8]), one obtains that

$$
\psi^{2}\left(x, t_{1}\right) \leq C\left(1+\frac{1}{t_{1}-t_{0}}\right) \int_{\Omega \times\left(t_{0}, t_{1}\right)} \psi^{2}(x, t)
$$


with the constant $C$ independent of $t$. It follows from Lemma 3.1 and the above inequality that

$$
\left|\frac{\partial f}{\partial t}\right|^{4} \leq C \int_{\Omega}|\tau(g)-V(g)|^{4} .
$$

Therefore we get a uniform bound for $\left|\frac{\partial f}{\partial t}\right|$. Now we have

$$
\Delta d(f(\cdot, t), g(\cdot)) \geq-C
$$

where the constant $C$ comes from the uniform bound of $\left|\frac{\partial f}{\partial t}\right|$ and the vector field $V$ by (2.2) and the parabolic system (3.1). A direct corollary of the inequality $(3.7)$ is that the distance function $d(f(\cdot, t), g(\cdot))$ has a uniform bound which is independent of $t$. Indeed applying the weak maximum principle to the elliptic inequality (3.7) one gets

$$
\sup _{\Omega} d(f(\cdot, t), g(\cdot)) \leq \sup _{\partial \Omega} d(f(\cdot, t), g(\cdot))+C|\Omega|^{\frac{1}{m}} .
$$

Consider the Dirichlet problem for the inhomogeneous Laplace equation in $\Omega$

$$
\begin{cases}\Delta u=-C, & \text { in } \Omega \\ u=0, & \text { on } \partial \Omega,\end{cases}
$$

where the constant comes from (3.7).

Let $u$ be the solution of problem (3.8), then

$$
\Delta\{d(f(\cdot, t), g(\cdot))-u(\cdot)\} \geq 0 .
$$

Hence

$$
\sup _{\Omega}\{d(f(\cdot, t), g(\cdot))-u(\cdot)\} \leq \sup _{\partial \Omega}\{d(f(\cdot, t), g(\cdot))-u(\cdot)\} .
$$

Therefore

$$
d(f(\cdot, t), g(\cdot)) \leq u(\cdot) .
$$

The solution of the problem (3.8) is of class $C^{2, \alpha}$ if the boundary $\partial \Omega$ smooth enough. So we have

$$
u(x) \leq C d(x, \partial \Omega) .
$$

This implies

$$
d(f(\cdot, t), g(\cdot)) \leq C d(x, \partial \Omega) .
$$


Take $\nu_{1}, \cdots, \nu_{m}$ to be a local orthonomal frame at $z_{0} \in \partial \Omega$ with $\nu_{1}, \cdots, \nu_{m-1}$ the tangent vectors of $\partial \Omega$ and $\nu_{m}$ the normal direction of the boundary. At the point $z_{0} \in \partial \Omega$ we then have

$$
\frac{\partial f(\cdot, t)}{\partial \nu_{j}}=\frac{\partial g(\cdot)}{\partial \nu_{j}}, \quad 1 \leq j \leq m-1,
$$

by the boundary condition, and

$$
\left|\frac{\partial f(\cdot, t)}{\partial \nu_{m}}\right| \leq C
$$

because of (3.10). Therefore we obtain a uniform bound for the gradient of the maps $f(\cdot, t)$, i.e.,

$$
e(f)(x) \leq C, \quad x \in \partial \Omega
$$

where the constant $C$ is independent of $t$.

From the above argument we know there is a constant $R$ so that

$$
d(f(\cdot, t), g(\cdot)) \leq R \quad \text { on } \Omega .
$$

Let $F=2 R^{2}-d^{2}(f(\cdot, t), g(\cdot))$, then $F \geq R^{2}$. Set

$$
A(x, t)=\frac{e(f)(x, t)}{F^{2}} .
$$

We have

$$
\left(\Delta-\frac{\partial}{\partial t}\right) A(x, t)=\frac{\left(\Delta-\frac{\partial}{\partial t}\right) e(f)}{F^{2}}-\frac{2 e(f)\left(\Delta-\frac{\partial}{\partial t}\right) F}{F^{3}}-\frac{4 \nabla e(f) \cdot \nabla F}{F^{3}}+\frac{6 e(f)|\nabla F|^{2}}{F^{4}} .
$$

By the Bochner formula, (cf [6], Ch. 8),

$$
\begin{aligned}
\left(\Delta-\frac{\partial}{\partial t}\right) e(f)= & \left\langle\left(\Delta-\frac{\partial}{\partial t}\right) \frac{\partial}{\partial x_{i}} f, \frac{\partial}{\partial x_{i}} f\right\rangle \\
= & \left\langle\nabla_{f_{*} \nu_{i}} V, f_{*} \nu_{i}\right\rangle+\left\langle\nabla_{\nu_{i} \nu_{j}} f, \nabla_{\nu_{i} \nu_{j}} f\right\rangle \\
& -\left\langle R^{N}\left(f_{*} \nu_{i}, f_{*} \nu_{j}\right) f_{*} \nu_{i}, f_{*} \nu_{j}\right\rangle+\left\langle f_{*} \operatorname{Ric}^{M} \nu_{i}, f_{*} \nu_{i}\right\rangle \\
\geq & |B(f)|^{2}-C e(f),
\end{aligned}
$$

where $B(f)=\nabla_{\nu_{i} \nu_{j}} f$ is the Hessian of the maps $f(\cdot, t)$. On the other hand,

$$
\begin{aligned}
\left(\Delta-\frac{\partial}{\partial t}\right) F & =\nabla_{N}^{2} F\left(f_{*} \nu_{i}, f_{*} \nu_{i}\right)-\left\langle\nabla_{N} F, \tau(f)-\frac{\partial f}{\partial t}\right\rangle \\
& =\nabla_{N}^{2} F\left(f_{*} \nu_{i}, f_{*} \nu_{i}\right)-\left\langle\nabla_{N} F, V\right\rangle .
\end{aligned}
$$


By Jacobi field estimates, (see, e.g. [6], Ch. 4)

$$
\nabla_{N}^{2} F\left(f_{*} \nu_{i}, f_{*} \nu_{i}\right) \leq-2 e(f)
$$

Hence

$$
\left(\Delta-\frac{\partial}{\partial t}\right) F \leq-2 e(f)+2 R|V|
$$

Here we have used the property that $\left|\nabla_{N} F\right|=2 d(f(\cdot, t), g(\cdot)) \leq 2 R$. Returning to (3.11), we have

$$
\left(\Delta-\frac{\partial}{\partial t}\right) A(x, t) \geq \frac{4}{F^{3}} e^{2}(f)-\frac{C}{F^{2}} e(f)-\frac{4 R|V|}{F^{3}} e(f)+I,
$$

where

$$
I=\frac{1}{F^{2}}|B(f)|^{2}-\frac{4}{F^{3}} \nabla e(f) \cdot \nabla F+\frac{6 e(f)}{F^{4}}|\nabla F|^{2} .
$$

Because

$$
\nabla A=\frac{F \nabla e-2 e \nabla F}{F^{3}}
$$

we have

$$
I=\frac{1}{F^{2}}|B(f)|^{2}-\left.\frac{2}{F^{3}}\left|\nabla e(f) \cdot \nabla F+\frac{2 e(f)}{F^{4}}\right| \nabla F\right|^{2}-\frac{2 \nabla A \cdot \nabla F}{F} .
$$

Notice that

$$
|B(f)|^{2}-\left.\frac{2}{F}\left|\nabla e(f) \cdot \nabla F+\frac{2 e(f)}{F^{2}}\right| \nabla F\right|^{2} \geq(|B(f)|-|d f||\nabla F|)^{2},
$$

we have

$$
I \geq-\frac{2 \nabla A \cdot \nabla F}{F}
$$

Therefore

$$
\left(\Delta-\frac{\partial}{\partial t}\right) A(x, t) \geq \frac{4}{F^{3}} e^{2}(f)-\frac{C}{F^{2}} e(f)-\frac{4 R|V|}{F^{3}} e(f)-\frac{2 \nabla A \cdot \nabla F}{F} .
$$

Or

$$
\left(\Delta-\frac{\partial}{\partial t}\right) A(x, t) \geq 4 F A^{2}-C A-\frac{4 R|V|}{F} A-\frac{2 \nabla A \cdot \nabla F}{F} .
$$

Let

$$
A(t)=\max _{x \in \bar{\Omega}} A(x, t)
$$


and assume that $A(t)=A\left(x_{t}, t\right)$ for some point $x_{t}$. If $x_{t} \in \partial \Omega$ for some $t$, we have

$$
e(f)(x, t) \leq 2 R^{2} A(x, t) \leq 2 R^{2} A\left(x_{t}, t\right) \leq 2 e(f)\left(x_{t}, t\right) .
$$

It follows from (3.11) that

$$
e(f)(x, t) \leq 2 C
$$

where the constant $C$ is the same as in (3.11). On the other hand if $x_{t} \in \Omega$, we have

$$
-\left.\frac{\partial}{\partial t} A\right|_{\left(x_{t}, t\right)} \geq 4 F A^{2}-C A-\frac{4 R|V|}{F} A
$$

Without loss of generality, we may assume that $\left.\frac{\partial}{\partial t} A\right|_{\left(x_{t}, t\right)} \geq 0$. Hence

$$
4 F A^{2}(t)-C A(t)-\frac{4 R|V|}{F} A(t) \leq 0 .
$$

That is to say

$$
A(x, t) \leq \frac{C}{4 F}+\frac{R|V|}{F^{2}}
$$

Therefore

$$
e(f)(x, t) \leq C R^{2}+R\|V\|,
$$

where $\|V\|=\max _{y \in N}|V(y)|$.

Up to now, we have proved

Theorem 3.1: Let the vector field $V$ satisfy the condition (3.2) with $\mu \leq$ $\frac{3}{4} \lambda(\Omega)$ for the first eigenvalue $\lambda(\Omega)$ of the Laplacian in $\Omega$. If the maps $f(\cdot, t)$ satisfy the parabolic system (3.1) for $0 \leq t<T$, then there is a constant $C$ which is independent of $t$ so that

$$
|d f(\cdot, t)|, \quad\left|\frac{\partial f}{\partial t}\right| \leq C
$$

By linearizing and using the theory of linear parabolic systems and the implicit function theorem, one gets from (3.17) that (3.1) has a solution for all of $t$. That is

Corollary 3.1: Let the vector field $V$ satisfy the condition (3.2) with $\mu \leq$ $\frac{3}{4} \lambda(\Omega)$ for the first eigenvalue $\lambda(\Omega)$ of the Laplacian in $\Omega$, then the parabolic system (3.1) has a solution for all time $t \in[0, \infty)$. Moreover, the solution of the parabolic system (3.1) has a uniform $C^{2, \alpha}$ bound. 
The last claim of Corollary 3.1 means that any sequence $t_{k} \rightarrow \infty$ will contain a subsequence $t_{n^{\prime}} \rightarrow \infty$ so that $f\left(\cdot, t_{k^{\prime}}\right)$ converges to a map $f$ in $C^{2}$. This leads to the existence for the Dirichlet problem

$$
\left\{\begin{array}{l}
\tau(f)=V(f) \\
f(x)=g(x), \quad x \in \partial \Omega
\end{array}\right.
$$

under a somewhat stronger condition than Theorem 3.1.

Theorem 3.2: Let the vector field $V$ satisfy the condition (3.2) with $\mu \leq \frac{3}{4} \lambda(\Omega)-\varepsilon$ for the first eigenvalue $\lambda(\Omega)$ of the Laplacian in $\Omega$, then the Dirichlet problem (3.18) has a solution.

Proof: By the same calculation as in the proof of Lemma 3.1, one gets that

$$
-\frac{1}{2} \frac{\partial}{\partial t} \int_{\Omega}\left|\frac{\partial f}{\partial t}\right|^{4} \geq\left.\left.\frac{3}{2} \int_{\Omega}|\nabla| \frac{\partial f}{\partial t}\right|^{2}\right|^{2}-\left(\frac{3}{2} \lambda(\Omega)-2 \varepsilon\right) \int_{\Omega}\left|\frac{\partial f}{\partial t}\right|^{4} .
$$

The Poincaré inequality gives

$$
\frac{\partial}{\partial t} \int_{\Omega}\left|\frac{\partial f}{\partial t}\right|^{4} \leq-4 \varepsilon \int_{\Omega}\left|\frac{\partial f}{\partial t}\right|^{4}
$$

That is to say

$$
\int_{\Omega}\left|\frac{\partial f}{\partial t}\right|^{4} \leq C_{0} \exp \{-4 \varepsilon t\} .
$$

Hence, for a sequence $t_{k}$ with $f\left(\cdot, t_{k}\right)$ convergent in $C^{2}$,

$$
\lim _{t_{k} \rightarrow \infty} \frac{\partial f}{\partial t}\left(x, t_{k}\right)=0 .
$$

Therefore the limit $f$ of $f\left(\cdot, t_{k}\right)$ will solve the problem (3.18).

Acknowledgement: The first author would like to express his gratitude to the Max Planck Institute for Mathematics in the Sciences for support and excellent working conditions.

\section{References.}

[1] Q.Chen, Maximum principles, uniqueness and existence for harmonic maps with potential and Landau-Lifshitz equations. Calc. Var. Partial Differential Equations 8 (1999), no. 2, 91-107. 
[2] W. Y. Ding, Y. D. Wang, Harmonic maps of complete noncompact Riemannian manifolds. Internat. J. Math. 2 (1991), no. 6, 617-632.

[3] A. Fardoun, A. Ratto, R. Regbaoui, On the heat flow for harmonic maps with potential. Ann. Global Anal. Geom. 18 (2000), no. 6, 555-567.

[4] J. Jost, Nonpositive curvature: geometric and analytic aspects. Birkhauser, 1997.

[5] J. Jost, Partial Differential Equations, GTM, Springer, New York, 2002.

[6] J. Jost, Riemannian Geometry and Geometric Analysis, Third Ed. Springer, Berlin Heidelberg, 2002.

[7] J. Jost, S.T. Yau, A nonlinear elliptic system for maps from Hermitian to Riemannian manifolds and rigidity theorems in Hermitian geometry. Acta Math. 170 (1993), no. 2, 221-254.

[8] J. Moser, A Harnack inequality for parabolic differential equations. Comm. Pure Appl. Math. 171964 101-134.

[9] X. W. Peng, G. F. Wang, Harmonic maps with a prescribed potential. C. R. Acad. Sci. Paris Ser.I Math. 327 (1998), no. 3, 271-276.

[10] R. Schoen, S. T. Yau, Compact group actions and the topology of manifolds with nonpositive curvature. Topology 18 (1979), no. 4, 361-380.

[11] W.von Wahl, The continuity or stability method for nonlinear elliptic and parabolic equations and systems. Proceedings of the Second International Conference on Partial Differential Equations (Italian) (Milan, 1992). Rend. Sem. Mat. Fis. Milano 62 (1992), 157-183(1994).

WenYi Chen

Department of Mathematics

WUHAN UNIVERSITY

Wuhan P. R. China 430072

JÜRgEN JOST

Max Planck Institute for Mathematics in the Sciences

INSELSTR. 22-26

D-04103 LEIPZIG

Germany 
